\title{
HIV Drug Resistance Among Children Initiating First-Line Antiretroviral Treatment in Uganda
}

\author{
Cissy Kityo,, Kim Catherina Eve Sigaloff,,3 Tamara Sonia Boender, ${ }^{2,3}$ \\ Elizabeth Kaudha, Joshua Kayiwa, 'Victor Musiime,, Andrew Mukuye,, Mary Kiconco," \\ Immaculate Nankya,' Llilian Nakatudde-Katumba, Job C.J. Calis, ${ }^{3,4}$ \\ Tobias F. Rinke de Wit, ${ }^{2,3}$ and Peter N. Mugyenyi ${ }^{1}$
}

\begin{abstract}
Background: There are limited data on primary human immunodeficiency virus drug resistance (HIVDR) in pediatric populations. This study aimed to assess the prevalence of primary HIVDR and associated risk factors among children initiating first-line antiretroviral therapy (ART) in Uganda.

Methods: At three Ugandan clinics, children (age $<12$ years) requiring ART were recruited between January 2010 and August 2011. Before starting ART, blood was collected for viral load and pol gene sequencing. Drug resistance mutations were determined using the 2010 International AIDS Society-USA mutation list. Risk factors for HIVDR were assessed with multivariate regression analysis.

Results: Three hundred nineteen HIV-infected children with a median age of 4.9 years were enrolled. Sequencing was successful in 279 children (87.5\%). HIVDR was present in $10 \%$ of all children and $15.2 \%$ of children $<3$ years. Nucleoside reverse transcriptase inhibitors (NRTIs), non-NRTI (NNRTI), and dual-class resistance was present in 5.7\%, 7.5\%, and 3.2\%, respectively. HIVDR occurred in $35.7 \%$ of prevention of mother-to-child transmission (PMTCT)-exposed children, 15.6\% in children with unknown PMTCT history, and $7.7 \%$ among antiretroviral-naive children. History of PMTCT exposure [adjusted odds ratio (AOR): 2.6, 95\% CI: 1.3-5.1] or unknown PMTCT status (AOR: 3.8, 95\% CI: 1.1-13.5), low CD4 (AOR: 2.2, 95\% CI: 1.33.6), current breastfeeding (AOR: 7.4, 95\% CI: 2.6-21), and current maternal ART use (AOR: 6.4, 95\% CI: 3.4-11.9) emerged as risk factors for primary HIVDR in multivariate analysis.

Conclusion: Pretreatment HIVDR is high, especially in children with PMTCT exposure. Protease inhibitor (PI)-based regimens are advocated by the World Health Organization, but availability in children is limited. Children with (unknown) PMTCT exposure, low CD4 count, current breastfeeding, or maternal ART need to be prioritized to receive PI-based regimens.
\end{abstract}

\section{Introduction}

$\mathbf{T}$ HE RAPID SCALE-UP of antiretroviral therapy (ART) regimens for human immunodeficiency virus (HIV), including prevention of mother-to-child transmission (PMTCT), in resource-limited countries is unprecedented. ${ }^{1-3}$ With increased access to PMTCT, the total number of children being born with HIV has significantly decreased. However, those children who do become infected after PMTCT failure are at particular risk of HIV drug resistance (HIVDR), as a result of non-nucleoside reverse transcriptase inhibitors (NNRTIs) used in maternal or pediatric drug regimens. ${ }^{4,5}$ Therefore, the World Health Organization (WHO) currently recommends initiating ART with a protease inhibitor (PI)based regimen in all children younger than 3 years. $^{6}$

In Uganda, it is currently estimated that 200,000 children are HIV infected. Of those, 70,000 (35\%) are on ART, with the remaining number expected to start ART in the near

\footnotetext{
${ }^{1}$ Joint Clinical Research Centre (JCRC), Kampala, Uganda.

${ }^{2}$ Amsterdam Institute for Global Health and Development, Amsterdam, the Netherlands.

${ }^{3}$ Department of Global Health, Academic Medical Center of the University of Amsterdam, Amsterdam, the Netherlands.

${ }^{4}$ Emma Children's Hospital, Academic Medical Centre, Amsterdam, the Netherlands.
}

(C) Cissy Kityo et al., 2016; Published by Mary Ann Liebert, Inc. This Open Access article is distributed under the terms of the Creative Commons Attribution Noncommercial License (<http://creativecommons.org/licenses/by-nc/4.0/>) which permits any noncommercial use, distribution, and reproduction in any medium, provided the original author(s) and the source are credited. 
future. Ugandan guidelines ${ }^{7,8}$ recommend providing ART to all HIV-infected children younger than 15 years regardless of CD4 cell count. NNRTI-based regimens are used, while for children younger than 2 years with reported PMTCT exposure, ritonavir-boosted lopinavir (LPV/r)-based regimens are preferred (in contrast to the WHO 2013 consolidated ART guidelines ${ }^{6}$ ) due to costs and limited availability of PIs. At the time of enrolling participants in this study, the national recommended choice of ART combinations ${ }^{9}$ was two nucleoside reverse transcriptase inhibitors (NRTIs) plus one NNRTI, either efavirenz or nevirapine, depending on CD4 count and $\mathrm{CD} 4 \%$ threshold for different age categories. Efavirenz was only prescribed to children older than 3 years. For infants exposed to PMTCT and since August 2010 for all PMTCTexposed children up to 24 months of age, ${ }^{10}$ a PI-based regimen was prescribed.

The prevalence and patterns of HIVDR with or without PMTCT exposure history in routine programs have not been well described. ${ }^{11}$ This is mainly due to the lack of laboratory facilities, affordability, and trained personnel for HIVDR testing. ${ }^{12,13}$ Available data in the sub-Saharan settings indicate that use of single-dose nevirapine (sdNVP), male gender, lower baseline immunological profiles, poor adherence, and breastfeeding are important predictors of HIVDR among children who have started on treatment. ${ }^{12-14}$ The data on factors associated with the presence of HIVDR besides previous exposure to antiretroviral (ARV) among children before ART initiation are sparse, ${ }^{15,16}$ yet baseline HIVDR is a critical indicator of the future success of ART as programs aim for universal access. The aim of this study was to evaluate the prevalence of and risk factors for primary HIVDR among newly diagnosed HIV-infected Ugandan children younger than 12 years. This was considered of particular importance in light of the relatively longer history of ART in Uganda compared to other African countries and the observed high baseline HIVDR prevalence of $13.8 \%$ in its adult population. ${ }^{17}$

\section{Materials and Methods}

\section{Study design and population}

Data reported here are based on the baseline assessments of children enrolled into the MARCH (Monitoring Antiretroviral Resistance in Children) study in Uganda. The study was conducted as a multicenter prospective observational cohort of HIV-1-infected children who initiated on ART from early 2010 to August 2011 at the Joint Clinical Research Centre (JCRC) study sites based in Kampala (central region), Fort Portal (western region), and Mbale (eastern region). The JCRC and its Regional Centers of Excellence are the main providers of HIV care and treatment in Uganda, with more than 20 years of experience in providing ART, training in HIV care, conducting clinical trials, and undertaking nationwide rollout of programs in ART care. Site and cohort characteristics have been described elsewhere. ${ }^{18}$

Potential participants were informed of the study and screened for eligibility by the study staff at each clinic. Children, aged up to 12 years who initiated ART, were included. Study participants were patients who attended the clinic as identified HIV-infected patients from the Early Infant Diagnosis (EID) national program that only started in late 2009. The older children were mainly self-referrals brought in by parents or guardians, referrals from other healthcare facilities, HIV-infected children from the Centers' diagnostic services, or children proactively sought by the Centers' community outreach programs. This program was intended to identify HIV-infected children who were not accessing existing ART programs and link them to treatment.

For those starting first-line regimens, a history of ART (i.e., three-drug regimen or mono/duo therapy) was an exclusion criterion. However, children with previous use of ARVs for PMTCT were included. Children who were failing on first-line therapy and initiating second-line therapy were included in the study, although they are not part of this current cross-sectional analysis. The ethical committees of the JCRC, the Uganda National Council for Science and Technology, and the Academic Medical Center of the University of Amsterdam approved the study protocol. The parent(s)/ guardian(s) of all eligible children provided written informed consent. Children older than 8 years who were aware of their HIV status provided written informed assent as per Ugandan research guidelines. Routine sociodemographic, clinical, and laboratory data were collected using electronic case report forms, which were aggregated in a Web-based data system. Whenever available, the health status and medication use of the mother were also captured.

\section{Laboratory procedures}

Routine laboratory results, including CD4 cell count and hemoglobin, were obtained from local laboratory records and/ or clinical notes. Before initiation of ART, an additional phlebotomy was performed, and EDTA-anticoagulated plasma specimens were stored at $-80^{\circ} \mathrm{C}$ and batch shipped to the JCRC reference laboratory in Kampala for determination of reference HIV RNA, as well as genotypic resistance testing on all specimens with HIV RNA above 1,000 copies/ml. For HIV RNA determination, the COBAS AmpliPrep/COBAS TaqMan HIV-1 test (Roche, Branchburg, NJ) was used. For HIV-1 genotyping, an in-house sequencing method encompassing the whole of protease and codons 1-300 of reverse transcriptase with a Beckman Coulter CEQ 8000 Analyzer (Beckman Coulter, Inc., Fullerton, CA) was used. ${ }^{19}$ Sequences were assembled and manually edited using BioEdit version 7.0. All final sequences were submitted to the ViroScore database (Advanced Biological Laboratories SA, France) for data storage. Drug resistance mutations (DRMs) were scored according to the 2010 International AIDS Society-USA list. ${ }^{20}$ Subtypes were determined using the SCUEAL HIV-1 subtyping tool ${ }^{21}$ and additional analysis with the REGA algorithm version $2.0^{22}$ once required.

\section{Statistical analyses}

Group comparisons for categorical data were performed using the chi-square or Fisher's exact tests and for continuous data using the Wilcoxon rank-sum test. Nutritional status was assessed by means of the WHO Child Growth Standards: WHO Anthro version 3.2.2 (age 0-5 years) and WHO Reference 2007 for height and weight (age 5-19 years). ${ }^{23-25}$ Immunodeficiency for age was classified according to the 2010 WHO guidelines; children were considered immunodeficient if they had a CD4 cell percentage $<25 \%$ for those younger than 5 years and a CD 4 cell count $<350$ cells $/ \mathrm{mm}^{3}$ for the ones older than 5 years. ${ }^{10}$ A second immunodeficiency threshold using 
total lymphocyte count (TLC) was based on the 2006 WHO guidelines using the following cutoffs: TLC $<4,000$ cells $/ \mathrm{mm}^{3}$ for those younger than 11 months, TLC $<3,000$ cells $/ \mathrm{mm}^{3}$ for those between 12 and 35 months, TLC $<2,500$ cells $/ \mathrm{mm}^{3}$ for those between 36 and 59 months, and TLC $<2,000$ cells $/ \mathrm{mm}^{3}$ for those older than 5 years. ${ }^{26}$

Univariate and multivariate logistic regression was performed to identify factors associated with the presence of at least one drug resistance mutation at initiation of first-line ART. Explanatory variables considered in the analysis were age, sex, previous ARV exposure (ARV naive, PMTCT exposed, or unknown), immunodeficiency for age, breastfeeding (past or current), maternal ART usage, maternal previous ARV use, WHO clinical stage, weight-for-age, height-forage $z$-scores, and HIV RNA load. Explanatory variables that were associated with the outcome variables $(p<.10)$ in univariate analysis were forwarded to the multivariate model using a step forward procedure. Biologically plausible interactions were examined. Results were expressed as ORs with $95 \%$ confidence intervals (CIs) and $p$ values, with $p<.05$ regarded statistically significant. Analyses were performed using the statistical software package STATA version 10 (STATA Corp LP, College Station, TX). All statistical inferential frameworks were based on the two-sided $p$ value, and statistical significance was based on the $5 \%$ error rate.

\section{Results}

Between January 2010 and August 2011, 372 children at three JCRC sites were enrolled into the study, of which 319 initiated first-line ART regimens. Recruited patients had a median age of 4.9 years [interquartile range: $2.3-9.0$ ], and half $(49.8 \%)$ were female. Most children had history of prior breastfeeding $(69.9 \%)$ or current breastfeeding at the time of screening $(8.2 \%)$. Current ART use was reported by 81 (28.3\%) mothers, and 17 (6.1\%) mothers had received drugs for PMTCT for previous pregnancies and/or pregnancy of the child who participated in the MARCH study. Most children $(83.5 \%)$ had no history of PMTCT exposure, and in $11.5 \%$ of children, PMTCT exposure was unknown. Of the children with unknown PMTCT exposure, 38\% had lost both their parents. Among children with reported PMTCT history $(n=14,5 \%)$, eight had received sdNVP and six had received extended-course prophylaxis. Thirty-seven (13.3\%) children were orphans, and $156(55.9 \%)$ had mothers as their primary caregivers. Most $(71.3 \%)$ patients had advanced disease (WHO stages 3 and 4) at screening, and CD4\% or cell count for age was below treatment threshold (24-59 months CD $4<750 /$ CD $4 \%<25 \%$ and $>5$ years CD $4 \leq 350$ ) among $60.6 \%$, according to the WHO 2010 guidelines. ${ }^{10}$

Eighteen children had a viral load $<1,000$ copies $/ \mathrm{ml}$ at baseline. HIV sequencing was successfully performed in 279 children (87.5\%, Fig. 1). At baseline, the prevalence of any DRM was 28/279 (10.0\%) among this population and 14/92 $(15.2 \%)$ among children $<3$ years of age. Baseline characteristics are summarized in Table 1, stratified by the presence of any DRMs. Most (53.8\%) patients had HIV-1 subtype A, $29.4 \%$ had HIV-1 subtype D, and $3.6 \%$ had HIV-1 subtype C or $\mathrm{G}$, yet $13.3 \%$ had circulating recombinant forms or unique recombinant forms.

Table S1 (Supplementary Data are available online at www.liebertpub.com/aid) and Figure 2 present the mutational

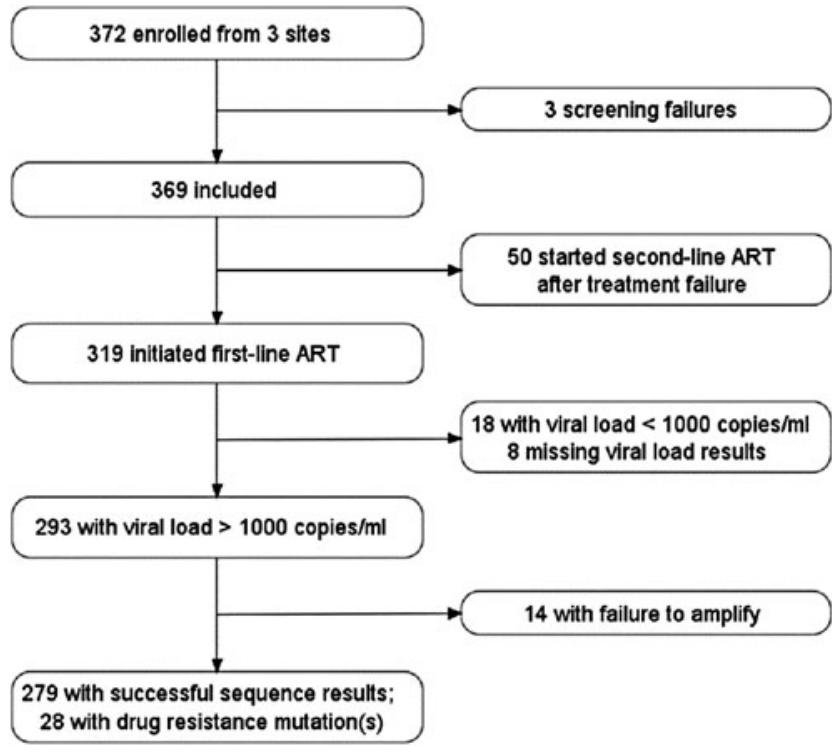

FIG. 1. Study flowchart. ART, antiretroviral therapy.

patterns among the enrolled patients initiating first-line ART stratified by previous ARV exposure. Overall, any mutation was found in $10.0 \%$ of children: In children with PMTCT exposure, with unknown PMTCT history, or who reported ARV naive, HIVDR was present in $35.7 \%, 15.6 \%$, and $7.7 \%$, respectively. In children younger than 3 years, the overall rate of any HIVDR mutation was $15.2 \%$ (14/92): In young children with PMTCT exposure, with unknown PMTCT history, or who reported ARV naive, HIVDR was present in $38.5 \%$ (5/ $13), 20 \%(2 / 10)$, and $10.1 \%$ (7/69), respectively. NRTI mutations were detected among 16/279 (5.7\%) patients-10 of whom were ARV-naive patients, three were PMTCT-exposed patients, and three patients had unknown ART exposure status. Thymidine analogue mutations were detected among 8/279 (2.9\%) patients - six of whom were ARV naïve, yet two had an unknown ART exposure. Most prevalent NRTI mutations found were M184V/I $(n=11)$ and K219Q $(n=4)$. NNRTI mutations were detected among $21 / 279(7.5 \%)$ patients-13 of whom were ARV-naive patients, four were PMTCT-exposed patients, and four patients had unknown ART exposure status. Most prevalent NNRTI mutations found were K103N $(n=11)$, Y181C $(n=9)$, and G190A $(n=6)$. Dual-class mutations were found among $9 / 279$ (3.2\%) patients.

Table 2 presents the demographic and clinical factors associated with HIVDR at initiation of first-line ART in a multivariate analysis. At multivariable level, factors, which significantly were associated with the presence of any DRMs, were previous ARV exposure, low CD4 count or percentage, breastfeeding status, and maternal ART status. Patients with prior PMTCT exposure were almost three times [adjusted OR (AOR): 2.6, 95\% CI: 1.3-5.1] more likely, yet patients with unknown ART exposure were almost four times (AOR: 3.8, 95\% CI: 1.1-13.5) more likely, to have any DRMs compared to those with no previous ART exposure, controlling for age, sex, CD4 count or percentage, breastfeeding status, and maternal ART status. Patients with CD4 count or percentages below treatment thresholds were twice (AOR: 2.2, 95\% CI: 1.3-3.6) more likely to have any DRMs compared to those 
Table 1. Baseline Characteristics of Children Initiating First-Line ART, Stratified by the Presence of any Drug Resistance Mutations

\begin{tabular}{|c|c|c|c|c|}
\hline & Total $(\mathrm{n}=279)$ & No $D R M(\mathrm{n}=251)$ & At least one DRM $(\mathrm{n}=28)$ & $\mathrm{p}$ \\
\hline Age-median [IQR] & $4.9[2.3-9.0]$ & $5.0[2.4-9.1]$ & $3.0[1.0-7.0]$ & $0.037^{\mathrm{a}}$ \\
\hline$<2$ years & $65(23.3)$ & $55(21.9)$ & $10(35.7)$ & $0.101^{b}$ \\
\hline$\geq 2$ years & $214(76.7)$ & $196(78.1)$ & $18(64.3)$ & \\
\hline Sex, female & $139(49.8)$ & $125(49.8)$ & $14(50.0)$ & $0.984^{\mathrm{b}}$ \\
\hline WHO clinical stage 3 or 4 & 199 (71.3) & $182(72.5)$ & $17(60.7)$ & $0.191^{\mathrm{b}}$ \\
\hline \multicolumn{5}{|l|}{ Weight-for-age $z$-score ${ }^{\mathrm{c}, \mathrm{d}}$} \\
\hline Underweight $(<-2$ SD) & $82(35.5)$ & $72(35.0)$ & $10(40.0)$ & $0.618^{\mathrm{b}}$ \\
\hline Severe $(<-3 \mathrm{SD})$ & $47(20.4)$ & $40(19.4)$ & $7(28.0)$ & $0.314^{\mathrm{b}}$ \\
\hline \multicolumn{5}{|l|}{ Height-for-age $z$-score ${ }^{\mathrm{c}, \mathrm{e}}$} \\
\hline Stunting $(<-2$ SD $)$ & $139(52.5)$ & $120(50.4)$ & $19(70.4)$ & $0.049^{\mathrm{b}}$ \\
\hline Severe $(<-3 \mathrm{SD})$ & $81(30.6)$ & 68 (28.6) & $13(48.2)$ & $0.036^{\mathrm{b}}$ \\
\hline CD4\% (in $<5$-year-olds) ${ }^{\text {f }}$ & 19 [11.8-27] & 19 [11.8-27] & $20[13.9-26]$ & $0.662^{\mathrm{a}}$ \\
\hline CD4 cell count (in $\geq 5$ year) & 362.5 [229-695] & 404 [249-695] & $169[56-506]$ & $0.076^{\mathrm{a}}$ \\
\hline $\begin{array}{l}\text { CD } 4 \% \text { or cell for age, below treatment } \\
\text { threshold }\end{array}$ & $106(39.4)$ & $99(40.7)$ & $7(26.9)$ & $0.171^{b}$ \\
\hline Viral load $\left(\log _{10}\right)$ & $5.2[4.6-5.6]$ & $5.1(4.6-5.6)$ & $5.4(4.6-5.8)$ & $0.459 \mathrm{a}$ \\
\hline \multicolumn{5}{|l|}{ Previous ARV exposure } \\
\hline None/ARV naive & $233(83.5)$ & $215(85.7)$ & $18(64.3)$ & $0.002^{\mathrm{b}}$ \\
\hline PMTCT $^{g}$ & $14(5.0)$ & 9 (3.6) & $5(17.9)$ & \\
\hline Unknown & $32(11.5)$ & $27(10.8)$ & $5(17.9)$ & \\
\hline \multicolumn{5}{|l|}{ Primary caregiver } \\
\hline Mother & $156(55.9)$ & $136(54.2)$ & $20(71.4)$ & $0.483^{\mathrm{h}}$ \\
\hline Father & $19(6.8)$ & $18(7.2)$ & $1(3.6)$ & \\
\hline Both parents died & $37(13.3)$ & $35(13.9)$ & $2(7.1)$ & \\
\hline Other & $67(24.0)$ & $62(24.7)$ & $5(17.9)$ & \\
\hline \multicolumn{5}{|l|}{ Breastfeeding } \\
\hline Yes, past & $195(69.9)$ & $181(72.1)$ & $14(50.0)$ & $<0.001^{\mathrm{b}}$ \\
\hline Yes, current & $23(8.2)$ & $15(6.0)$ & $8(28.6)$ & \\
\hline None or unknown & $61(21.9)$ & $55(21.9)$ & $6(21.9)$ & \\
\hline \multicolumn{5}{|l|}{ Mother current ART } \\
\hline First-line ART & $74(26.5)$ & $61(24.3)$ & $13(46.4)$ & $0.021^{\mathrm{h}}$ \\
\hline Second-line ART & $5(1.8)$ & $3(1.2)$ & $2(7.1)$ & \\
\hline None & $160(57.3)$ & $150(59.8)$ & $10(35.7)$ & \\
\hline Unknown & $40(14.3)$ & $37(14.7)$ & $3(10.7)$ & \\
\hline \multicolumn{5}{|l|}{ Mother previous ART } \\
\hline Yes, PMTCT & $17(6.1)$ & $15(6.0)$ & $2(7.1)$ & $0.041^{\mathrm{h}}$ \\
\hline Yes, ART & $3(1.1)$ & $1(0.4)$ & $2(7.1)$ & \\
\hline No & $139(50.0)$ & $124(49.4)$ & $15(53.6)$ & \\
\hline Unknown & $120(42.9)$ & $111(44.2)$ & $9(32.1)$ & \\
\hline \multicolumn{5}{|l|}{ HIV-1 subtype } \\
\hline A & $150(53.8)$ & $136(54.2)$ & $14(50.0)$ & $0.848^{\mathrm{h}}$ \\
\hline $\mathrm{D}$ & $82(29.4)$ & $74(29.5)$ & $8(28.6)$ & \\
\hline $\mathrm{C}$ or $\mathrm{G}$ & $10(3.6)$ & $9(3.6)$ & $1(3.6)$ & \\
\hline CRF or URF & 37 (13.3) & $32(12.8)$ & $5(17.9)$ & \\
\hline
\end{tabular}

Data presented as $n(\%)$ or median [interquartile range].

${ }^{\mathrm{a}}$ Wilcoxon rank-sum test.

${ }^{\mathrm{b}}$ Chi-square test.

${ }^{\mathrm{c}}$ Some $\%$ may not add up to $100 \%$ because of missing data for some participants.

${ }^{\mathrm{d}}$ Weight-for-age calculated for ages $0-10$ years only, $n=231$.

${ }^{\mathrm{e}}$ Height-for-age, $n=265$.

${ }^{\mathrm{f}} \mathrm{CD} 4 \%$ in $<5$-year-olds, $n=135$; CD4 count in $\geq 5$-year-olds, $n=134$; treatment threshold based on WHO 2010 guidelines.

${ }^{\mathrm{g}}$ PMTCT consisted of single-dose nevirapine $(n=7)$, an extended course of nevirapine $(n=6)$, sometimes in combination with zidovudine $(n=2)$, or was unknown $(n=1)$.

${ }^{\text {h}}$ Fisher's exact test.

ARV, antiretroviral; ART, antiretroviral therapy; CRF, circulating recombinant form; DRM, drug resistance mutation; PMTCT, prevention of mother-to-child transmission; SD, standard deviation; URF, unique recombinant form; WHO, World Health Organization.

with CD4 count or percentages above treatment thresholds, adjusting for age, sex, their previous ARV exposure, breastfeeding status, and maternal ART status.

Compared to patients who had been breastfed in the past, patients who were still being breastfed at the time of study were seven times (AOR: 7.4, 95\% CI: 2.6-21.0) more likely, yet patients whose breastfeeding status was unknown or who reported never to have breastfed were twice (AOR: 1.9, 95\% CI: 1.5-2.3) more likely, to have any DRMs, after controlling for age, sex, their previous ARV exposure, CD4 count or 


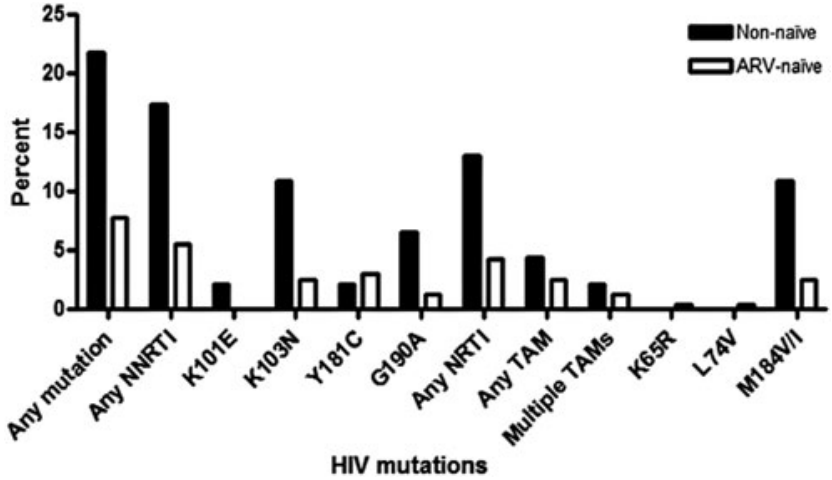

FIG. 2. Profile of specific antiretroviral drug resistance mutations detected among ART naïve and non naïve (PMTCT exposed and unknown exposure) children.

percentage, and maternal ART status. Patients whose mothers were currently on ART were six times (AOR: 6.4, 95\% CI: 3.4-11.9) more likely, whereas those whose mothers' current ART status was unknown or whose mothers were reported to be deceased were 1.4 times $(1.4,95 \%$ CI: $0.8-2.3)$ more likely, to have any DRMs compared to those whose mothers reported not to be taking any ART, after controlling for age, sex, CD4 count or percentage, breastfeeding status, and previous ARV exposure.

\section{Discussion}

This study assessed HIVDR mutations among Ugandan HIV-infected children $<12$ years of age initiating ART be- tween January 2010 and August 2011. Given the study setup, the overall rate of HIVDR was $10.0 \%$ among all children and $15.2 \%$ among children $<3$ years old, with age significantly associated with having at least one DRM.

The HIVDR rates in this study are much higher than previously reported rates of transmitted drug resistance (TDR) in Uganda and Cameroon for similar age groups ${ }^{27,28}$ and lower than recent rates shown in younger children in Kenya, South Africa, and Zimbabwe with much higher PMTCT exposure rates, although using HAART. ${ }^{11,14,29}$ As in adults, the TDR rate is expected to increase over time with increasing coverage and uptake of PMTCT. The finding of lower rates of HIVDR in a limited number of other African pediatric cohorts is explained by the fact that our cohorts represent relatively low percentages of children with previous PMTCT exposure (sdNVP). Moreover, HIVDR mutations might be archived ${ }^{30-32}$ and not be detectable with the RNA-based technology of genotyping used since the average age of children included in our studies is higher. The decline in mutations is a function of time since ART exposure and reported rates may be an underestimation.

Overall, a trend of increasing HIVDR was detected in children who were reportedly ART naive (7.7\%) versus those with unknown ART exposure (15.6\%) versus PMTCTexposed children (35.7\%). Although higher rates were observed, similar trends were seen in children $<3$ years of age $(92$ of 279 children overall), with rates of $10.1 \%, 20 \%$, and $38.5 \%$, respectively. These data support classifying children with unknown PMTCT exposure as PMTCT exposed. In addition, children with unknown exposure were more likely to be orphans, and ascertainment of PMTCT exposure was difficult.

Children who were currently breastfeeding or whose mothers were currently on ART at the time of enrollment

Table 2. Demographic and Clinical Factors Associated with HiVDR at Initiation of First-Line ART

\begin{tabular}{|c|c|c|c|c|c|c|}
\hline & \multirow{2}{*}{$\begin{array}{c}\text { No. of } \\
\text { sequences }\end{array}$} & \multirow[b]{2}{*}{ No. with any DRM } & \multicolumn{2}{|c|}{ Univariate analysis } & \multicolumn{2}{|c|}{ Multivariate analysis } \\
\hline & & & OR $(95 \% C I)$ & $\mathrm{p}$ & $O R(95 \% C I)$ & $\mathrm{p}$ \\
\hline \multicolumn{7}{|l|}{ Age } \\
\hline$\geq 5$ years & 138 & 9 & 1.0 & & & \\
\hline$<5$ years & 141 & 19 & $2.2(1.1-4.7)$ & 0.034 & & \\
\hline \multicolumn{7}{|l|}{ Sex } \\
\hline Female & 139 & 14 & 1.0 & & & \\
\hline Male & 140 & 14 & $1.0(0.5-2.0)$ & 0.983 & & \\
\hline \multicolumn{7}{|l|}{ Previous ARV exposure } \\
\hline None & 233 & 18 & 1.0 & & 1.0 & \\
\hline PMTCT exposed & 14 & 5 & $6.6(1.0-42.1)$ & 0.045 & $2.6(1.3-5.1)$ & 0.006 \\
\hline Unknown & 32 & 5 & $2.2(1.3-4.2)$ & 0.015 & $3.8(1.1-13.5)$ & 0.040 \\
\hline \multicolumn{7}{|l|}{ CD4 count or $\%$} \\
\hline Above treatment threshold & 106 & 7 & 1.0 & & 1.0 & \\
\hline Below treatment threshold & 163 & 19 & $1.9(1.5-2.3)$ & $<0.001$ & $2.2(1.3-3.6)$ & 0.002 \\
\hline \multicolumn{7}{|l|}{ Breastfeeding } \\
\hline Past & 195 & 14 & 1.0 & & 1.0 & \\
\hline Current & 23 & 8 & $6.9(1.7-27.5)$ & 0.006 & $7.4(2.6-21.0)$ & $<0.001$ \\
\hline None/unknown & 61 & 6 & $1.4(0.9-2.3)$ & 0.168 & $1.9(1.5-2.3)$ & $<0.001$ \\
\hline \multicolumn{7}{|l|}{ Maternal ART } \\
\hline None & 86 & 6 & 1.0 & & 1.0 & \\
\hline Current ART & 79 & 15 & $3.1(1.4-7.1)$ & 0.007 & $6.4(3.4-11.9)$ & $<0.001$ \\
\hline Mother deceased/unknown & 114 & 7 & $0.9(0.5-1.4)$ & 0.582 & $1.4(0.8-2.3)$ & 0.195 \\
\hline
\end{tabular}

Multivariate logistic regression with robust standard errors. Data are given as odds ratio (95\% CI). CD4 count or \% treatment threshold based on WHO 2010 guidelines. Maternal previous ARV use, WHO clinical stage, weight-for-age, height-for-age, and HIV RNA load at ART initiation not associated in univariate analysis.

CI, confidence interval; HIVDR, HIV drug resistance. 
were more likely to have DRMs. Children with no/unknown history of breastfeeding had higher odds of having baseline resistance compared to past history of breastfeeding; hence, this group of children should be considered as high risk for DRMs. These data also underscore the fact that taking breastfeeding history is difficult and not so reliable, especially in cases where the mother is deceased (26.5\%). The average age at study entry was 3 years for patients with at least one DRM versus 5 years for those without DRMs. This further supports classifying these categories of children as a broader definition of ART/PMTCT exposed as they are likely to respond better to PI-based regimens. The WHO ART guidelines $2013^{6}$ recommend lopinavir-based regimen for all children $<3$ years of age irrespective of PMTCT exposure. However, current Uganda guidelines ${ }^{8}$ and other developing countries still have NNRTI-based regimen as the preferred first-line treatment for children aged $<3$ years and may be exposing many children younger than 3 years with known and unknown ARV exposure to suboptimal therapy.

HIVDR in ARV-naive children was higher among children $<3$ years, with overall and NNRTI-specific mutation rates of $10.1 \%$ and $7.3 \%$, respectively, compared to $6.7 \%$ and $4.9 \%$ among children $\geq 3$ years. This rate is much lower compared to other settings, where NNRTI mutations were up to $24 \%$ in ART-naive children. ${ }^{11}$ High rates of NNRTI mutations were observed in children with unknown history of PMTCT, with $10 \%$ in children $<3$ years compared to $13.6 \%$ in children $\geq 3$ years. Among all children not exposed to PMTCT (none and unknown), NNRTI mutations were detected among $7.59 \%$ of children $<3$ years compared to $5.91 \%$ of children $\geq 3$ years who would be started on a suboptimal NNRTI-based ART regimen. These data provide more support for the WHO recommendations for empiric PI-based first-line ART in children $<3$ years.

While PI-based regimens are considered the most appropriate regimen for infants and young children by the WHO, providing these regimens to all infants and children $<3$ years in some resource-limited settings, such as Uganda, may be challenging. Currently, pediatric LPV/r is only available as a liquid formulation with a high alcohol content that tastes terrible with the potential for suboptimal adherence and requires cold chain until the point of dispensing. Pediatric HIV treatment is complicated, and dosing strategies change as the child grows from infancy to adolescence. Furthermore, LPV/r is costly, and administering this with TB treatment is complex. Efforts to overcome some of these obstacles include replacement of this liquid formulation with a more tolerable version that is palatable and does not require refrigeration. Possible formulations include sprinkles, minitabs, or granules that could be mixed with food or given with breast milk. ${ }^{33}$

The most common mutation, which was present in 3.9\% of the samples, was M184V, which confers resistance to the NRTI drugs lamivudine, emtricitabine, and abacavir but delays resistance to zidovudine and stavudine ${ }^{34,35}$ The most common NNRTI mutations were K103N (3.9\%), Y181C (2.9\%), and G190A (2.2\%), which confer resistance to efavirenz, nevirapine, and delavirdine. However, $3.2 \%$ of the children had dual-class resistance. These mutations are similar to what has been observed from other studies. ${ }^{11,27,29,36}$ These findings suggest that genotypic resistance is fairly common among HIV-infected children starting ART in Uganda and may adversely affect response to first-line treatment in addition to other risk factors, which are unique to children.
The study had some limitations. This study is different in approach from the newly recommended pediatric HIVDR surveys by the WHO. ${ }^{37}$ Specifically, the enrolled children were older (median age, 4.9 years) than recommended by the WHO ( $<18$ months) and are a survivor cohort since the majority of HIV children will die by age 2 years if not treated with ARV drugs. ${ }^{38}$ In addition, the majority of children recruited at this time were reportedly PMTCT unexposed (233/ $279,83.5 \%$ ), while this will be less likely the case in more recent pediatric HIV surveys when adoption of option $\mathrm{B}+$ for PMTCT is more widespread. Given the age group and PMTCT exposure, caution should be applied when directly comparing these results with (future) pediatric HIVDR surveys according to the new WHO guidelines.

We recruited older children than most studies that have evaluated TDR in children, and the observed HIVDR may be an underestimate of the actual rates due to archived DRMs. More sensitive assays may be able to help address this issue. We were not able to determine drug resistance in the mothers to link the DRMs to the resistance patterns in their children. With revised pediatric guidelines and expansion of EID programs, increasingly younger children will be recruited on treatment in the future. However, current ART access for children is only $35 \%$ in sub-Saharan Africa, ${ }^{39}$ and there are still many older children who are yet to be put on treatment in the coming years as per the 2013 WHO guidelines. These data are relevant for their management and selection of optimal ART regimens. The patients in the study were recruited before the era of intensive PMTCT, with the majority of women using either sdNVP or double therapy and limited access to HAART for their general care. However, the study was able to show that PMTCT, maternal ART, and breastfeeding are still important risk factors for TDR in this cohort.

The strength of the study is that this is an observational study that recruited participants who would normally be seeking for treatment at these ART centers, and therefore, these results are directly applicable to programs. Detailed demographic, clinical, and programmatic data were collected to determine TDR and related risk factors, and the study was conducted in at least three regions of the country making it more representative.

In conclusion, this study demonstrated that HIVDR is common, especially in HIV-infected children of younger age and those exposed to ART through PMTCT, maternal ART, and breastfeeding. Even children with unknown PMTCT exposure had high rates of DRMs. This implies that just taking history of PMTCT does not clearly delineate children at risk of having DRMs. The broader definition of PMTCT exposure needs to include those children with unknown history as well as those with mothers on ART and/or current breastfeeding who need to be prioritized to receive PI-based regimens according to the new WHO guidelines.

In our setting, children without risk factors may still respond well to regimens without PIs, which is more practical to implement than PI-based regimens. Follow-up data from this cohort will show what the true outcome is of these children.

This study was done before the shift to option $\mathrm{B} / \mathrm{B}+$, and our results cannot predict resistance in this group. However, with rollout of option $\mathrm{B}+$, the total number of infections should decrease with proper taking of drugs, and breakthrough infections possibly due to nonadherence of the mother may have 
more DRMs. However, when vertical transmission takes place in the presence of ARV drugs, the risk of HIVDR increases, as shown in this study. This highlights the need for in-depth evaluation and more extensive surveys among the infected children in the era of expanded PMTCT. The availability of these data at relevant times would significantly inform clinical management and promote improved quality of life.

\section{Acknowledgments}

The authors thank all study participants and their caregivers, doctors, nurses, and support staff at three JCRC participating sites, staff at the AIGHD, and the PharmAccess Foundation.

Joint Clinical Research Centre: Annet Nandudu, James Nkalubo, Isaac Egau, and Lincoln Mugarura from JCRC Kampala; Michael Owor, Christine Matama, and Florence Nambaziira from JCRC Fort Portal; and Mary Abwola, Fred Senono, Ronald Namisi, and Sylivia Nakusi from JCRC Mbale.

AIGHD and PharmAccess: Cees Hesp and John Dekker from PharmAccess; Corry Manting from AIGHDAmsterdam and Cathy Nalubwama from AIGHD_Kampala.

Funding: The MARCH study is an initiative of JCRC and PharmAccess Foundation and was funded by the European \& Developing Countries Clinical Trials Partnership (EDCTP) EEIG TA 08_40200_022. The funders had no role in the study design, data collection, data analysis, data interpretation, decision to publish, or writing of the report.

GenBank accession numbers: All HIV-1 pol sequences in this study have been deposited in the GenBank under the following accession numbers: KT347880-KT348210.

\section{Author Contributions}

C.K. is the principal investigator. C.K., T.F.R.d.W., P.N.M., K.C.E.S., and J.C.J.C. designed the study and developed the protocol. C.K., L.N.K., E.K., V.M., A.M., and M.K. established the cohort and supervised data collection. J.K. and S.T.B. supervised data management. C.K., K.C.E.S., J.K., and T.F.R.d.W. analyzed the data, interpreted it, and drafted the article. All authors reviewed and approved the final version of the article.

\section{Author Disclosure Statement}

No competing financial interests exist.

\section{References}

1. Chi BH, Adler MR, Bolu O, et al.: Progress, challenges, and new opportunities for the prevention of mother-to-child transmission of HIV under the US President's Emergency Plan for AIDS Relief. J Acquir Immune Defic Syndr 2012;60 Suppl 3:S78-S87.

2. Gibb DM, Giaquinto C: Children with HIV infection: Special cases. Lancet 2000;356 Suppl:s34.

3. World Health Organization, Unicef, UNAIDS: Global update on HIV treatment 2013: Results, impact and opportunities. WHO Bull 2013(June):7. ISBN 9789241505734.

4. Paredes R, Marconi VC, Lockman S, Abrams EJ, Kuhn L: Impact of antiretroviral drugs in pregnant women and their children in Africa: HIV resistance and treatment outcomes. J Infect Dis 2013;207 Suppl 2:S93-S100.
5. Ton Q, Frenkel L: HIV drug resistance in mothers and infants following use of antiretrovirals to prevent motherto-child transmission. Curr HIV Res 2013;11:126-136.

6. WHO. (World Health Organization). Consolidated guidelines on the use of antiretroviral drugs for treating and preventing HIV infection. WHO, Geneva, Switz. 2013;(June): 94-95,109,32-33,90,146-153.

7. Alex A: The national antiretroviral treatment guidelines for Uganda 2013. ATIC Newsl 2014(March):1-12.

8. Uganda Ministry of Health. Addendum to the national antiretroviral treatment guidelines. 2013(December):24 .

9. Uganda Ministry of Health. National antiretroviral treatment guidelines for adults, adolescents, and children. 2009(June):1-80. www.aidstar-one.com/sites/default/files/ treatment/national_treatment_guidelines/Uganda_guidelines_ 2009.pdf

10. WHO. (World Health Organization). Antiretroviral therapy for HIV infection in infants and children: towards universal access. WHO, Geneva, Switz. 2010.

11. Kuhn L, Hunt G, Technau K-G, et al.: Drug resistance among newly diagnosed HIV-infected children in the era of more efficacious antiretroviral prophylaxis. AIDS 2014;28: 1673-1678.

12. Kamya MR, Mayanja-Kizza H, Kambugu A, et al.: Predictors of long-term viral failure among ugandan children and adults treated with antiretroviral therapy. J Acquir Immune Defic Syndr 2007;46:187-193.

13. Sebunya R, Musiime V, Kitaka SB, Ndeezi G: Incidence and risk factors for first line anti retroviral treatment failure among Ugandan children attending an urban HIV clinic. AIDS Res Ther 2013;10:25.

14. Lel R, Ngaira J, Lihana R, Khamadi S: HIV-1 drug resistance mutations among infants born to HIV-positive mothers in Busia, Kenya. AIDS Res Hum Retroviruses 2014;30: 1236-1238.

15. Kébé $\mathrm{K}$, Bélec L, Diop Ndiaye $\mathrm{H}$, et al.: The case for addressing primary resistance mutations to non-nucleoside reverse transcriptase inhibitors to treat children born from mothers living with HIV in sub-Saharan Africa. J Int AIDS Soc 2014;17:18526.

16. Kumar M: Prevalence of drug resistance in human immunodeficiency virus type 1-infected treatment-naive children in Pune, India. AIDS Res Human Retroviruses 2014;30:294-298.

17. Hamers RL, Wallis CL, Kityo C, et al.: HIV-1 drug resistance in antiretroviral-naive individuals in sub-Saharan Africa after rollout of antiretroviral therapy: a multicentre observational study. Lancet Infect Dis 2011;11:750-759.

18. Boender TS, Sigaloff KCE, Kayiwa J, et al.: Barriers to initiation of pediatric HIV treatment in Uganda: A mixedmethod study. AIDS Res Treat 2012;2012:817506.

19. Richard N, Juntilla M, Abraha A, et al.: High prevalence of antiretroviral resistance in treated Ugandans infected with non-subtype B human immunodeficiency virus type 1 . AIDS Res Hum Retroviruses 2004;20:355-364.

20. Johnson VA, Brun-Vézinet F, Clotet B, et al.: Update of the drug resistance mutations in HIV-1: December 2010. Top HIV Med 2010;18:156-163.

21. Kosakovsky Pond SL, Posada D, Stawiski E, et al.: An evolutionary model-based algorithm for accurate phylogenetic breakpoint mapping and subtype prediction in HIV-1. PLoS Comput Biol 2009;5:e1000581.

22. de Oliveira T, Deforche K, Cassol S, et al:: An automated genotyping system for analysis of HIV-1 and other microbial sequences. Bioinformatics 2005;21:3797-3800. 
23. de Onis M, Onyango AW, Borghi E, Siyam A, Nishida C, Siekmann J: Development of a WHO growth reference for school-aged children and adolescents. Bull World Health Organ 2007;85:660-667.

24. Onyango AW, de Onis M, Caroli M, et al:: Field-testing the WHO child growth standards in four countries. J Nutr 2007; 137:149-152.

25. WHO. WHO child growth standards: Length/height-for-age, weight-for-age, weight-for-length, weight-for-height and body mass index-for age, WHO, OMS. http://apps.who.int/ bookorders/anglais/detart 1 .jsp?sesslan $=1 \&$ codlan $=1 \&$ codcol $=$ 15\&codcch=660, accessed February 12, 2015.

26. WHO. WHO|antiretroviral therapy of HIV infection in infants and children. www.who.int/hiv/pub/paediatric/infants/ en/ (2006), accessed February 12, 2015.

27. Sánchez PR, Holguín A: Drug resistance in the HIV-1infected paediatric population worldwide: A systematic review. J Antimicrob Chemother 2014;69:2032-2042.

28. Fokam J, Salpini R, Santoro MM, et al.: Drug resistance among drug-naive and first-line antiretroviral treatmentfailing children in Cameroon. Pediatr Infect Dis J 2011;30: 1062-1068.

29. Zimbabwe MOH: Surveillance of initial drug resistant HIV-1 among children under 18 months of age newly diagnosed with HIV survey report. 2012 (December):1-23.

30. Loubser S, Balfe P, Sherman G, Hammer S, Kuhn L, Morris L: Decay of K103N mutants in cellular DNA and plasma RNA after single-dose nevirapine to reduce motherto-child HIV transmission. AIDS 2006;20:995-1002.

31. Martinson NA, Morris L, Gray G, et al.: Selection and persistence of viral resistance in HIV-infected children after exposure to single-dose nevirapine. J Acquir Immune Defic Syndr 2007;44:148-153.

32. Hunt GM, Coovadia A, Abrams EJ, et al: HIV-1 drug resistance at antiretroviral treatment initiation in children previously exposed to single-dose nevirapine. AIDS 2011; 25:1461-1469.

33. Musiime V, Fillekes Q, Kekitiinwa A, et al.: The pharmacokinetics and acceptability of lopinavir/ritonavir minitab sprinkles, tablets, and syrups in african HIV-infected children. J Acquir Immune Defic Syndr 2014;66:148-154.
34. Miller V, Ait-Khaled M, Stone C, et al: HIV-1 reverse transcriptase (RT) genotype and susceptibility to RT inhibitors during abacavir monotherapy and combination therapy. AIDS 2000;14:163-171.

35. Turner D, Brenner BG, Routy JP, Petrella M, Wainberg MA: Rationale for maintenance of the M184v resistance mutation in human immunodeficiency virus type 1 reverse transcriptase in treatment experienced patients. New Microbiol 2004;27 (2 Suppl 1):31-39.

36. Penazzato M, Apollo T, Dzangare J, et al. World Health Organization HIV drug resistance surveillance in children less than 18 months newly diagnosed with HIV in Zimbabwe. In: IAS 2013-Kuala LumPur, MalaysIa 30th June 3rd July.; 2013:Poster N15_DR.

37. Bertagnolio S, Penazzato M, Jordan MR, Persaud D, Mofenson LM, Bennett DE: World Health Organization generic protocol to assess drug-resistant HIV among children $<18$ months of age and newly diagnosed with HIV in resource-limited countries. Clin Infect Dis 2012;54 Suppl 4:S254-S260.

38. Newell M-L, Coovadia H, Cortina-Borja M, Rollins N, Gaillard P, Dabis F: Mortality of infected and uninfected infants born to HIV-infected mothers in Africa: A pooled analysis. Lancet 2004;364:1236-1243.

39. UNAIDS. 2013 UNAIDS report on the global AIDS epidemic. UNAIDS, 2013. Available at www.unaids.org/en/ resources/documents/2013/name,85053,en.asp.f; www.unaids .org/sites/default/files/media_asset/20131219_AccessART AfricaStatusReportProgresstowards2015Targets_en_0.pdf (2013), accessed February 12, 2015.

Address correspondence to:

Cissy Kityo

Joint Clinical Research Centre (JCRC)

Plot 101 Lubowa

PO Box 10005

Kampala

Uganda

E-mail: ckityo@jcrc.org.ug; ckityo@yahoo.com 